\title{
BMJ Open Northern Manhattan Hispanic Caregiver Intervention Effectiveness Study: protocol of a pragmatic randomised trial comparing the effectiveness of two established interventions for informal caregivers of persons with dementia
}

José A Luchsinger, ${ }^{1}$ Louis Burgio, ${ }^{2}$ Mary Mittelman, ${ }^{3}$ Ilana Dunner, ${ }^{4}$ Jed A Levine, ${ }^{5}$ Jian Kong, ${ }^{6,7}$ Stephanie Silver, ${ }^{6,7}$ Mildred Ramirez,, ${ }^{6,7}$ Jeanne A Teresi ${ }^{6,7}$

To cite: Luchsinger JA, Burgio L, Mittelman $\mathrm{M}$, et al. Northern Manhattan Hispanic Caregiver Intervention Effectiveness Study: protocol of a pragmatic randomised trial comparing the effectiveness of two established interventions for informal caregivers of persons with dementia. BMJ Open 2016;6:e014082. doi:10.1136/bmjopen-2016014082

- Prepublication history for this paper is available online. To view these files please visit the journal online (http://dx.doi.org/10.1136/ bmjopen-2016-014082).

Received 30 August 2016 Accepted 4 November 2016

CrossMark

For numbered affiliations see end of article.

Correspondence to Dr José A Luchsinger; jal94@cumc.columbia.edu

\section{ABSTRACT}

Introduction: The prevalence of dementia is increasing without a known cure, resulting in an increasing number of informal caregivers. Caring for a person with dementia results in increased stress and depressive symptoms. There are several behavioural interventions designed to alleviate stress and depressive symptoms in caregivers of persons with dementia with evidence of efficacy. Two of the best-known interventions are the New York University Caregiver Intervention (NYUCl) and the Resources for Enhancing Alzheimer's Caregivers Health (REACH). The effectiveness of the NYUCI and REACH has never been compared. There is also a paucity of data on which interventions are more effective in Hispanics in New York City. Thus, we proposed the Northern Manhattan Hispanic Caregiver intervention Effectiveness Study (NHiCE), a pragmatic clinical trial designed to compare the effectiveness of adaptations of the NYUCl and the REACH in informal Hispanic caregivers of persons with dementia in New York City.

Methods and analysis: NHiCE is a 6-month randomised controlled trial comparing the effectiveness of adaptations of the NYUCI and REACH among 200 Hispanic informal adult caregivers of persons with dementia. The planned number of sessions of the NYUCI and REACH are similar. The primary outcome measures are changes from baseline to 6 months in the Zarit Caregiver Burden Scale and Geriatric Depression Scale. Our primary approach to analyses will be intent-to-treat. The primary analyses will use mixed random effects models, and a full information maximum likelihood approach, with sensitivity analyses using generalised estimating equation.

Ethics and dissemination: NHiCE is approved by the Institutional Review Board of Columbia University Medical Center (protocol AAAM5150). A Data Safety Monitoring Board monitors the progress of the study. Dissemination will include reports of the

\section{Strengths of limitations of this study}

Northern Manhattan Hispanic Caregiver intervention Effectiveness Study will be first study to compare the effectiveness of the New York University Caregiver Intervention (NYUCl) and Resources for Enhancing Alzheimer's Caregivers Health (REACH) interventions in any population.

- The study is a randomised controlled trial.

- The outcome data will be collected independent of the interventionists.

- The study will last 6 months, measuring shortterm, but not long-term effects.

- The study will have limited power for subgroup analyses.

characteristics of the study participants, as well as a report of the results of the clinical trial.

Trial registration number: NCT02092987, Preresults.

\section{INTRODUCTION}

Dementia is a syndrome characterised by impairment of memory and other cognitive abilities as well as behavioural symptoms, severe enough to impair the ability to live independently. ${ }^{1}$ The most common cause of late onset dementia is Alzheimer's disease, ${ }^{23}$ comprising $\sim 70 \%$ of cases, but vascular and mixed dementias are also common, comprising up to $25 \%$ of cases. ${ }^{4}$ Dementia prevalence increases after the age of 70 years ${ }^{5}$ and may reach $50 \%$ in persons 85 years and older. ${ }^{6}$ In 2013, the Alzheimer's Association estimated that 5.2 million people ( 1 in 8 elders) have Alzheimer's dementia (AD) cared for by 15.4 million unpaid caregivers, 
including relatives and friends, resulting in US $\$ 214$ billion in annual costs. ${ }^{3}$ A recent publication from the Research and Development (RAND) corporation reported that dementia is the most expensive condition to care of, more expensive than even heart disease or cancer, including the costs attributed to the time spent in informal caregiving by relatives. ${ }^{7}$ Despite increased understanding of dementia, no preventive or curative measure exists, ${ }^{8}$ and results of trials of new agents are discouraging. ${ }^{9}$ Consequently, the numbers of caregivers burdened by dementia will continue to increase. Hispanics, the fastest growing ethnic group in the $\mathrm{USA}^{10}{ }^{10}$ is also the group with the fastest growing number of dementia cases. ${ }^{11}$ Dementia prevalence in Hispanics is several times higher than in Non-Hispanic Whites (NHW) nationally $(27.9 \%$ vs $10.9 \%$ in persons aged $75-$ 84 years; $62.9 \%$ vs $30.2 \%$ in persons 85 years and older) ${ }^{3}$ and in New York City. ${ }^{12} 13$

The care of persons with dementia is challenging. ${ }^{14-16}$ These individuals require intense supervision and care, risking caregivers' psychological, physical ${ }^{17-19}$ and financial health. ${ }^{3}$ Caregiver stress leads to premature nursing home placement (NHP) for the person with dementia, ${ }^{19}{ }^{20}$ but caregivers report emotional and physical stress even after NHP. ${ }^{21}{ }^{22}$ Most caregivers report no guilt after NHP, but this is less common in Hispanics, ${ }^{3} 23$ who traditionally delegate less care of affected relatives to paid caregivers. ${ }^{23}$ A 2011 nationwide telephone survey conducted by the Alzheimer's Association highlights Hispanic caregivers' unique characteristics. They are younger than NHW and Non-Hispanic Black (NHB); ${ }^{3}$ less likely to be married than NHW; more likely to have children or grandchildren under age 18 in their household than NHW and NHB; more likely to be a primary caregiver than NHW and Asian-Americans (AA); more likely to make $<$ US\$50 000 annually than NHW and AA and more likely to need help balancing work and family and finding personal time than NHW. ${ }^{3}$ Small studies show Hispanics experience more strain and less social support than other racial/ethnic groups, ${ }^{24} 25$ despite extensive social networks, ${ }^{26}$ and less acculturated Hispanic caregivers experience more depression. ${ }^{27}$ In summary, Hispanic caregivers of a person with dementia experience more depression and higher burden due to unique cultural characteristics. Thus, our project focuses on Hispanics.

Two of the best-known and tested dementia caregiver interventions are the New York University Caregiver Intervention (NYUCI) ${ }^{28-30}$ and the Resources for Enhancing Caregivers' Health (REACH). ${ }^{31}{ }^{32}$ However, the acceptability and effectiveness of the NYUCI and the REACH have never been compared. REACH has demonstrated efficacy among Cuban Hispanics in South Florida and Mexican Hispanics in California in a randomised controlled trial (RCT). ${ }^{31}$ However, this trial was not sufficiently powered to test differential efficacy in these Hispanic subgroups and did not include Dominicans or Puerto Ricans, the most numerous
Hispanic subgroups in New York City. The NYUCI has demonstrated efficacy in NHW spouse caregivers, and more recently in NHW adult children caregivers in Minnesota, ${ }^{33}$ but does not have evidence of efficacy from a clinical trial in Hispanics.

Most dementia caregiving research, including NYUCI, REACH and its translation, REACH OUT, has been guided by Pearlin's stress process model (SPM) ${ }^{34}$ The SPM proposes that caregiving context variables affect each part of the stress process and can have implications for the types of stressors facing caregivers. It also posits that the perception or appraisal of those stressors and social support are mediators that can affect outcomes such as caregiver depression and physical health. These mediators can be affected in positive ways by the REACH and NYUCI.

\section{New York University Caregiver Intervention}

The NYUCI was evaluated in a longitudinal RCT over more than two decades that included 406 spouse caregivers $\left(89 \%\right.$ NHW sample) ${ }^{35}$ The intervention alleviated the deleterious effects of caregiving on the mental and physical health of spouse caregivers and postponed or prevented NHP of their spouses with $\mathrm{AD}$. Moreover, the intervention's effects on caregiver depression were long-lasting and continued through NHP and death of the person with $\mathrm{AD} .{ }^{29} 36$ The underlying theme of the NYUCI is that improving social support improves the caregiver's ability to withstand the difficulties of caregiving and obviates or defers the need for NHP. Changes in caregiver reaction to the spouse's memory and behavioural problems, satisfaction with social support and depression collectively explained $61.2 \%$ of the intervention's effect on NHP of their spouses. A mediation analysis demonstrated that a substantial proportion of effect on change in these outcomes could be attributed to intervention-induced increases in the caregivers' satisfaction with their social support networks. ${ }^{37}$ The intervention increased objective indicators of social support and more subjective measures. The effects of change in satisfaction with social support were found to be significant predictors of change in stress appraisals $(p<0.0001)$ and change in depression $(p<0.0001)$. Results of a recently published study demonstrate the effectiveness in of the NYUCI in postponing NHP among persons whose caregivers are adult children. ${ }^{33}$ The NYUCI is listed and described in detail on the National Registry of Evidence-based Programs and Practices website.

\section{Resources for Enhancing Alzheimer's Caregiver Health}

REACH I was an National Institutes of Health (NIH)-funded Cooperative Agreement where six intervention sites investigated six different intervention packages with a common outcomes assessment battery. The REACH group published outcomes from each site ${ }^{38}$ as well as a meta-analysis of findings across the six sites. ${ }^{39}$ In REACH I, Burgio and colleagues used a single 
treatment intervention. In this study, an equal number of NHB and NHW spouse and adult child caregivers were trained in behaviour management and general problem-solving skills. ${ }^{40}$ The intervention was effective in reducing behaviour problems in care recipients ${ }^{41}$ This was also the first study to show a differential response to a caregiver intervention based on race and relationship factors. ${ }^{40}$ The findings of the meta-analysis ${ }^{39}$ showed that the most efficacious interventions were multicomponent, action-oriented and were conducted in-home. On the basis of these results the investigators designed what is known as REACH II. This intervention was tested using a multisite RCT. The REACH II intervention was an intensive tailored caregiver intervention that employed multiple components to address multiple needs of caregiver (spouses/children)-care-receiver dyads. The driving therapeutic approach was problem solving. Equal numbers of NHB, NHW and Hispanics were recruited into the study. There was significant improvement in a composite measure of quality of life compared to no treatment controls in all ethnic groups. ${ }^{31}$ REACH OUT is the most recent, less intensive adaptation of REACH that has been implemented and tested in community settings and will be used in this study. The North Carolina REACH II Translation Project (contract number 90AE0325t) was funded by the Administration on Aging (AoA) and conducted from 10/01/08 to 09/30/11. This was an attempt by the North Carolina Division of Aging \& Adult Service (NCDAAS) to implement the REACH II RCT treatment protocol with complete fidelity. However, the RCT protocol was shown to be too intensive for practical use in the community. All aspects of the project, including the number and duration of sessions, breadth of the outcome assessment and treatment implementation battery, and complexity of the intervention were beyond the scope of a community service organisation, in this case the Agencies on Aging in North Carolina. Consequently, in 2010 NCDAAS applied for and received funding from AoA to examine the translated REACH OUT protocol (2010-2013). Also in 2010, AoA funded the State Agencies on Aging of Vermont and Puerto Rico to implement REACH OUT for their elders and their caregivers. REACH is listed and described in detail on the National Registry of Evidence-based Programs and Practices website. REACH OUT is listed as an evidence-based treatment on the Alliance for Caregiving and Roselyn Carter Center for Caregiving websites.

\section{Comparison of NYUCI and REACH}

We chose to test the NYUCI and REACH OUT because they are the better known and more widely recommended caregiver interventions with established efficacy $^{42} 43$ as demonstrated by their support by the AoA and their acknowledgement in a recent report by the Alzheimer's Association. ${ }^{32}$ NYUCI and REACH OUT have important similarities: (1) they are based on the same conceptual model, (2) are designed to ameliorate caregiver depression and subjective stress/burden, (3) involve direct interaction between the interventionist and the caregiver/family, (4) the interventions focus on similar skills/topics during intervention and (5) both allow tailoring of the intervention to the needs of the caregiver/family. However, they differ on two characteristics that prior research has found to be potent factors in interventions: therapeutic technique and mode of delivery. REACH OUT focuses on skills training through the use of action-oriented formal problem solving, goal setting, written action plans, homework assignments and therapist tracking. NYUCI focuses on reducing negative family interactions, improving family support of the primary caregiver and helping the caregiver to understand and react more effectively to the behaviour of the person with dementia. The second difference is in the mode of delivery-and it is one of degree. Although the Hispanic version of REACH OUT encourages family involvement, it is still primarily an intervention focusing on a primary caregiver. NYUCI requires that caregiver and family or friends participate in counselling sessions. Finally, although both interventions are tailored, the tailoring corresponds with the content and the therapeutic technique (eg, styles of counselling) for the caregiver/family in the NYUCI, based initially on the intake assessment and modified in response to the needs and desires of the primary caregiver and family, as revealed in the counselling sessions; whereas REACH OUT uses a standardised risk assessment at intake to choose which skill areas are emphasised.

\section{Study aims, hypothesis and design}

The primary research question of the Northern Manhattan Hispanic Caregiver intervention Effectiveness Study (NHiCE) is which of two of the best-known dementia caregiver interventions, the NYUCI or REACH OUT, is more effective in alleviating depressive symptoms and caregiver burden among Hispanic caregivers in New York City. Our primary aim and our statistical plan is to compare the effectiveness of the NYUCI and REACH OUT. In our secondary aims, we will explore the predictors of effectiveness for each. These secondary analyses will provide practical information on who benefits the most from each intervention.

We hypothesise that the NYUCI will be more effective than REACH OUT in reducing caregiver depressive symptoms and burden among Hispanics because of its focus on family-centred counselling, which is posited to be more important among Hispanic caregivers because of a cultural emphasis among Hispanics on family interactions in interventions.

\section{METHODS AND ANALYSIS}

In order to answer our primary question, we are conducting a pragmatic randomised trial comparing the NYUCI versus REACH OUT in 200 Hispanic caregivers of persons with dementia in the New York City. The 
total time of the intervention is 6 months. We have powered and designed the study to answer the primary question. However, we have additional questions that could also be of importance to caregivers that we have termed exploratory because we do not know if our study is powered to answer those questions, although we may be. These questions include whether the NYUCI and REACH OUT have differential effectiveness on caregiver stress and physical health, and whether there are caregivers who benefit more from the NYUCI or REACH OUT depending on their characteristics such as family position and acculturation level.

\section{Rationale for study design}

NHiCE is funded by the Patient-Centered Outcomes Research Institute (PCORI). We took into consideration the PCORI draft methodology report in deciding which study design to use. $^{44}$ This included randomised trials and non-randomised studies. Since there has never been a comparison of the NYUCI and REACH OUT, we decided to conduct a randomised trial. We consider this trial as pragmatic because we do not have the strict inclusion criteria of the efficacy studies of the NYUCI and the REACH and because we are conducting the study in a community setting partnering with stakeholders in a way that resembles how the interventions would be implemented in real life. For example, we do not limit participants to caregivers of persons with Alzheimer's disease only but include caregivers of persons with any type of dementia. We also include any relative caregiver, not only spouses or adult children. Our experience in prior work with a similar population has taught us that this sample is the most representative of Hispanic caregivers in New York City. These other types of caregivers were not included in the prior evaluation of the REACH, which included only spouses and adult children. Increasingly, active control (comparison) groups are included in RCTs, ${ }^{45}$ and comparative effectiveness trials, including observational studies ${ }^{46}$ are becoming commonplace. Our view is that randomised clinical trials remain a method of choice, when feasible; ${ }^{47}$ however, analytic methods that include examination of 'dose received' should be considered. ${ }^{48}$ Our design is to compare two evidence-based interventions that have not been compared head-to-head among Hispanic caregivers. An active comparison group that receives the REACH OUT intervention will be included in this study, with one treatment group (NYUCI). We framed the study as a superiority trial of NYUCI over REACH.

\section{Study population and participants}

The sampling frame for this study is the Hispanic community of New York City, primarily from Northern Manhattan. The main catchment areas of Columbia University Medical Center (CUMC), where the study is based, are Washington Heights-Inwood (WH) and Central Harlem, which comprise Northern Manhattan. WH begins at 155th Street and extends northward to the tip of Manhattan Island. WH is bounded on the west by the Hudson River and on the east by East River. WH is predominantly Hispanic with $75.2 \%$ of the population reporting that they were of Hispanic origin in the 2000 Census. WH has the largest Dominican community in the USA ( $10 \%$ of the total). Dominicans comprise about $75 \%$ of Hispanics in WH. The other Hispanic subgroups in $\mathrm{WH}$ are Puerto Ricans, Mexicans, Cubans and Ecuadorians who make up $8 \%, 3 \%, 3 \%$ and $2 \%$ of $\mathrm{WH}$ Hispanics. Central Harlem, spanning East-West from the Harlem River to Morningside Avenue, and North and South from 155th Street to 110th Street in Manhattan, also has a growing Hispanic population now exceeding $25 \%$. The most recently updated community health profile for $\mathrm{WH}$ shows that $32 \%$ of the adults in the community rate their own health as fair or poor, significantly higher than the citywide average of $21 \%$. Fewer have health insurance, with $33 \%$ un-insured or only partially covered, compared to $27 \%$ citywide. One-third (32\%) also have no primary care provider, compared to $24 \%$ citywide, and among immigrants, $37 \%$ do not have a regular provider. Depression and severe mental illness are greater problems in $\mathrm{WH}$ than in the rest of the city. Dementia and cognitive impairment in general are also higher in Hispanics in this population compared to NHW in Northern Manhattan.

All study participants are adult informal caregivers of a person with a clinical diagnosis of dementia. An additional requirement for participation is that, in each family, the person with dementia or the caregiver has to have at least one relative or close friend living in the New York City metropolitan area. The caregiver must be emotionally and physically capable of participating. Caregivers with clinical depression that is treated can participate in the study. Those not treated will be referred for treatment. Caregivers with depression with psychotic features or suicidal ideation will not be eligible to participate and will be referred for treatment if not under treatment. Caregivers with other severe psychiatric illnesses such as schizophrenia are not be eligible to participate. The full list of inclusion criteria is listed below.

\section{Inclusion criteria}

- Self-identified Hispanic.

- Between the ages of 18 and 90 .

- Caregiver is related to persons receiving care either as a spouse (including common law partners) or a blood or in-law relative.

- Person receiving care has been diagnosed with dementia and reports at least one memory/cognition and one daily functioning symptom at our screening questionnaire.

- Caregiver is physically able to provide care.

- Caregiver does not have a diagnosis of major psychiatric disorder other than depression.

- Caregiver does not have severe depression characterised by a score of $\geq 20$ on the on the Patient Health Questionnaire-9 (PHQ-9). 
- Caregiver does not have depression with psychotic features or suicidal ideation or attempts in the last 5 years.

- Caregiver is expected to live in New York City in the next 12 months (or willing to do the interview in NYC).

- There is at least one relative or close friend living in the New York City Metropolitan Area (New York, New Jersey, Connecticut, USA).

- Caregiver is not currently receiving counselling for caregiving issues on a regular basis.

- Care recipient is not in a nursing home.

- Caregiver will not be travelling for $>2$ weeks during the study period.

- Hearing is sufficient to allow for communication.

\section{Exclusion criteria}

- Not Hispanic.

- Caregiver is a formal paid caregiver.

- Person receiving care does not have dementia.

- Other than depression, caregiver has a major psychiatric disorder such as schizophrenia.

- Severe depression characterised by a score of $\geq 20$ on the PHQ-9.

- Caregiver has depression with psychotic features or suicidal ideation in the last 5 years.

\section{Recruitment and retention strategies}

Our recruitment strategy is based on previous experience $^{49}$ and have been approved by the CUMC Institutional Review Board. Our target recruitment is 200 participants in 24 months or between 8 and 9 participants a month. The study supports outreach personnel at community partners who help with recruitment. All study personnel are fluent in English and Spanish. Recruitment sources include:

- Community-based and hospital-based medical and mental health practices in New York City.

- Churches.

- Senior centres.

- The modes of outreach include

- Talks at churches and senior centres in English and/or Spanish by Dr Luchsinger.

- Monthly emails sent to medical and mental health practitioners.

- Talks at physician group practices.

- Bilingual study flyers and brochures.

- Paid ads in church newsletters.

- Free ads in the newsletter of the Alzheimer's Association.

Retention strategies include:

- Reimbursement for time and transportation.

- Conduct of study procedures and interventions at the time and place of preference of participants. Time may include evenings and weekends.

- Birthday cards.

- Holiday cards.

\section{Study arms}

There are two arms in the study, the NYUCI and the REACH OUT. The NYUCI and REACH OUT have six visits in 6 months of similar duration in addition to ad hoc visit contacts in those 6 months followed by ad-hoc contacts only after 6 months and after month 12. Thus, an important premise of this study is that the two interventions have similar 'doses' and only the mode of delivery is different. In addition to the specific NYUCI and REACH OUT interventions, all participants are referred to a dedicated social worker for an intake interview for social work needs, such as medical insurance, help with housing. This social worker is based at our community partner, Riverstone Senior Life Services.

\section{Summary of NYUCI intervention}

The first component consists of two individual and four family counselling sessions that include relatives or close friends suggested by the caregiver. The content of these sessions is determined by the needs of each caregiver and other participating family members (eg, learning techniques for management of troublesome patient behaviour, and promoting communication among family members). These sessions last between 1 and 1.5 hours. The second component of the intervention is participation in a support group to provide the caregiver with continuous emotional support and education. The third component of the treatment is 'ad hoc' counsellingthe continuous availability of counsellors to caregivers and families to help them deal with crises and with the changing nature and severity of their relatives' symptoms over the course of the disease. The emergence of new psychiatric and behavioural problems of patients, which are generally more stressful than the need for assistance with activities of daily living or physical limitations, often precipitate ad hoc calls from caregivers. Ad hoc counselling makes it possible for caregivers and families to determine the amount of contact they have with the counsellors beyond the scheduled structured sessions. Ad hoc counselling will be available during the 12 months of subject participation.

The NYUCI is delivered by a bilingual (English, Spanish) social worker with experience in dementia and caregiver issues.

\section{Summary of the REACH OUT intervention}

All aspects of the REACH OUT intervention involve problem-solving techniques and the development of written action plans. The goal of this intervention is to engage the caregiver in joint problem solving with the objective of creating a written action plan targeting specific caregiving problems (eg, improving caregiver health, resolving dangers in the physical environment and care recipient problem behaviours). Problem-solving strategies are used to generate relevant information about the 'target problem' and the overall caregiving situation, with special emphasis placed on the context in which the target problem occurs. In general, problem solving 
should be thought of as a 'mindset' or guiding strategy to use when working with the caregiver to develop and modify action plans over the intervention period. The basic steps of problem solving are as follows: (1) define the problem, (2) set goals, (3) brainstorm with caregiver and list possible solutions on a pad of paper, (4) select solutions, (5) develop an action plan based on these solutions and (6) implement the action plan, track progress and make adjustments as needed. The first step in REACH OUT is to use a risk appraisal to determine how much emphasis to place on each of the intervention components. Thus, the intervention is standardised with respect to the components available but varies with respect to the dosing or depth of treatment delivered for each component. The tailoring of the intervention is guided by the findings of the risk appraisal.

There are six specific skill sets taught to the caregivers: (1) education about dementia, caregiving and stress; (2) helping the caregiver stay healthy; (3) keeping the home safe for the dementia patient; (4) teaching the caregiver how to maintain emotional well-being though relaxation, (5) behaviour management and (6) enhancing social support. The basic delivery elements of the intervention are as follows: intervention occurs over 6 months; there are six in-home sessions, $\sim 1-1.5$ hours in length, similar to the NYUCI. Under exceptional conditions, up to 2 in-home sessions can be substituted by therapeutic telephone sessions (at least $30 \mathrm{~min}$ with a maximum duration of $90 \mathrm{~min}$ ); caregivers receive a Caregiver Notebook that contains educational information about dementia, self-care, safety and other relevant caregiver issues. The Caregiver Notebook will also include copies of all action plans. The Caregiver Notebook serves as a tool for organising intervention materials and a resource guide for the caregiver to use during and after the intervention; the Case Manager reviews specific issues related to dementia, caregiving and stress using education material provided in the Caregiver Notebook; the counsellor provides individualised action plans to address targeted problems such as care recipient behaviours, caregiver health behaviours and social support.

The counsellor teaches caregivers how to enhance their emotional well-being. This is performed by teaching relaxation techniques and, if indicated, pleasant events training. The REACH Counsellor will have similar qualifications to the NYUCI counsellor.

\section{Justification for duration, treatment dose and adherence} The NYUCI $^{36}$ and REACH ${ }^{31}$ were tested as 6-month intervention in efficacy studies. In the case of the NYUCI, the efficacy studies included an ad-hoc intervention period after the original 6 months. The adaptations of both that are currently being implemented also last 6 months. The NYUCI and REACH last 6 months and have an equal number of study visits. Thus, it seems reasonable to postulate that the dose of both interventions is comparable. However, we will measure intervention fidelity and ad-hoc contact with the therapists for each intervention during the 6-month intervention period in order to ensure that both interventions are comparable. If there are differences, we will adjust for these differences in post hoc analyses and report these results.

\section{Randomisation}

Following consent, determination of eligibility and completion of baseline measures, the coordinator will alert the data coordinating center electronically either via encrypted email or data uploads to a secure server. Respondents will be randomised 1:1 to comparison treatment groups. The randomisation algorithm accommodates rolling enrolment, and the results are checked periodically for balance.

\section{Rationale for choice of outcomes and outcome measures}

The original assessment used by the NYUCI and REACH included scales to measure burden. The NYUCI also used a measure of depression that is used in NHiCE. The primary outcomes are caregiver depression and burden. Secondary outcomes include caregiver health, measured with the caregiver physical health form ${ }^{50}$ and the Patient Reported Outcomes Measurement Information System (PROMIS) Physical Health Scale, ${ }^{51-53}$ and caregiver stress measured by the Perceived Stress Scale. ${ }^{54}$ Potential covariates include the Revised Memory and Problem Behaviour Checklist ${ }^{55}$ the Stokes Social Network Scale ${ }^{56}$ and an assessment of the severity of patient dementia, the Global Deterioration Scale. ${ }^{57}$ Acculturation and type of caregiver (spouse or daughter) may be important moderator variables that will be informative in descriptive analyses identifying which interventions work for which subgroups of individuals.

Although there are numerous measures of depression, the selection of the Geriatric Depression Scale (GDS) as the primary measure of depression was based on the use of this measure in studies of the NYUCI, and findings from the literature related to differential item functioning (DIF) in measures of depression. Although there are few studies of DIF among Latino samples, our review ${ }^{58}$ of DIF in depression measures showed that many Center for Epidemiologic Studies Depression Scale (CES-D) ${ }^{59}$ items were biased for ethnically diverse groups. Thus, we decided to use the GDS, ${ }^{60}$ which although also containing items with DIF, has fewer such items and less with somatic content. The latter have been found to be problematic with older individuals with comorbidities; ${ }^{61}$ we expected that such individuals would likely comprise a large part of the caregiver sample. We also use the shortform depression measure from the NIH PROMIS item bank. ${ }^{62}{ }^{63}$ This measure has been found to be relatively DIF-free ${ }^{64}$ with low magnitude of DIF in Latino elders. ${ }^{65}$ Owing to its primacy in studies of depression, it was included as an exploratory end point measure.

The Zarit Burden Interview (ZBI) measures dementia caregiver burden and collapse ${ }^{66}$ and has evidenced acceptable reliability. The ZBI has been shown to 
improve in Hispanic communities in South Florida in the REACH intervention. ${ }^{316768}$ Since an objective of the NYUCI and REACH is to alleviate caregiver burden, we chose to include a measure of caregiver burden previously used in Hispanics in the REACH study and in the RCTs of the NYUCI as a coprimary outcome.

\section{Rationale for examining heterogeneity of treatment effects}

Owing to low power for subgroup analyses, heterogeneity of treatment (HTE) effects will be treated as descriptive as defined in the methods guidelines for PCORI. ${ }^{44}$ Subgroups examined will include type of caregiver (spouse, daughter) and level of acculturation, measured using the Bidimensional Acculturation Scale for Hispanics. ${ }^{69}$ As behaviours and attitudes of the 'majority culture' blend with those of the 'minority culture' via the process of acculturation, a shift towards individualism might be observed, potentially impacting the familistic norms to provide elder care.

\section{Analytic methods}

It is expected that those assigned to the NYUCI intervention will, on average, exhibit greater 6-month decreases in ZBI and GDS than those assigned to the REACH condition. The relative effectiveness of the NYUCI will be examined in order to determine whether the intervention does more good than harm relative to the REACH as provided in the usual circumstances found in the community. ${ }^{46}$ Our primary approach to analyses will be intent-to-treat (ITT). Although ITT is not optimal under certain circumstances, for example, high levels of nonadherence, other analytic approaches, for example, as treated, result in bias associated with limited causal inference due to compromised random assignment. Depending on the level of missing data, analytic strategies discussed below can be used in comparative effectiveness research to address non-compliance, for example. ${ }^{70}$ The primary analyses will use mixed random effects models, and a full information likelihood (FIML) approach, with sensitivity analyses using generalised estimating equation (GEE). ${ }^{71}$ The change from pretreatment to post-treatment values of continuous outcomes will be modelled as functions of baseline values, treatment and the interaction of baseline and treatment. A general longitudinal mixed effects model using SAS PROC MIXED will be used to allow for the possible group heterogeneity in residual variances and serial correlations that may require modelling to satisfy model assumptions and improve model fit.

Depending on the observed correlation between the dependent variables, MANOVA will be performed in sensitivity analyses and MANCOVA in subgroup analyses. A significant interaction term for one of the groups would indicate that the effect of one of the treatments is different for ZBI and GDS; in that case, two treatment effects will be estimated for each outcome. If the interaction is not significant, a model with only main effects for the outcomes and treatment will be fit and the treatment effect (common for ZBI and GDS) will be estimated from this model. In addition to significance testing, we will estimate the treatment effects with 95\% CI. Bartlett's test of sphericity will inform about the degree of intercorrelation among the outcome measures in order to determine suitability of the basic MANOVA model.

\section{Heterogeneity of treatment effect}

As specified in the methodology report to PCORI, because there is an interest in examining subgroups to help determine for which individuals interventions may be effective, we will perform descriptive HTE by adding the potential effect modifiers, acculturation and type of caregiver into the MANCOVA model as interaction terms. Thus, the model described above will be changed to $\mathrm{k}_{\mathrm{c}}=3$ exogenous baseline covariates that include terms for group, time by group and main effects and interaction terms for acculturation and type of caregiver (spouse, child).

\section{Bias and missing data}

Randomisation should obviate the need for covariates in the primary analyses of effectiveness of the NYUCI. However, selection and attrition bias and failures of randomisation may occur. If one or more sources of potential bias are identified, the predicted values from those analyses will be included as covariates in sensitivity analyses. Depending on the degree of attrition bias, another approach is to perform propensity score analyses, in which the treatment groups are combined, and a logistic regression predicting original group membership from covariates performed. The resulting probabilities are then arrayed in quintiles, and the participants within each quintile randomly assigned to new groups. The analyses will be re-run with the new group designations in order to determine if the effects were similar in the new analyses with groups equalised.

\section{Treatment of missing data}

Using the above-described maximum-likelihood approach to estimate treatment effects, we will include the baseline data for these participants in the analysis. Under the assumption that the missing data are either missing completely at random (MCAR) or missing at random (MAR), this method, in conjunction with the covariate to adjust for attrition bias (if necessary), yields ITT parameter estimates that are consistent with what would be expected if there were no missing data. Although it is anticipated that there will be little missing data, if large amounts of missing data are observed, methods such as multiple imputation or propensity score matching will be considered.

\section{Intervention dose}

Sample size permitting, exploratory analyses will be conducted so as to investigate the impact of differential participation, stratifying the participants in the treatment conditions based on their degree of participation and examining differences between strata on the outcome 
measures at follow-up. We will examine the subset of the intervention group that followed the protocol. Specifically, a dose-response of the intervention will be examined in several ways: continuous, binary (received or did not receive the intervention) and ordinal. The designations for the binary and ordinal variables will be determined based on clinical judgement about what constitutes completion and varying levels of completion. SAS Proc Mixed will be used for the dose-response analysis of the ordinal (categorical) number of sessions attended. ANCOVA will be used for the primary outcomes, adjusting for baseline values, within the intervention group. We will also create an ordinal variable of levels of adherence with the control group as the reference group. Although the benefits of randomisation are lost to some extent, this approach will inform about dose-response.

\section{Analyses and sample size calculation}

The sample size calculations are based on the number of participants needed to provide adequate power to test the primary hypothesis related to group differences in depression and burden at 6-month follow-up. The primary power calculations assume separate analyses of burden and depression measures; however, the use of MANOVA to perform a simultaneous test was also considered because it is generally more powerful and makes use of more information. In addition, although FIML estimation procedures will be used for the primary analyses, thereby allowing us to include participants who do not complete the follow-up assessment (on an ITT basis), the power calculations include scenarios in which there is loss to follow-up as large as $20 \%$. Effect size estimates (Cohen's $d^{72}$ ) for depression and burden were obtained from prior studies of caregivers.

\section{Assumptions for power calculations}

The estimate used in the power calculations for $\alpha$ (reliability) was 0.85 and for $\mathrm{r}$ (correlation between time 1 and time 2) between 0.50 and 0.70 . The estimate used for $\sigma$ (pooled SD) was $\approx 9.8$ for the Zarit and 7.47 for the GDS (table 1). A SAS macro was used for power calculations.

\section{Power for primary analyses}

In addition to standard power calculations to examine group differences in end point measures (shown in figures 1 and 2), also examined were different scenarios regarding correlations between baseline and 6-month follow-up outcome measures.

The following formula from Fleiss (ref. 73, pp. 4-5) was modified to include different scenarios related to correlations between the two waves of data: ${ }^{74}$ $n *=\left(4(1-\rho)\left(\sigma^{2}\right)\left(\mathrm{Z}_{\alpha / 2}+Z_{\beta}\right)^{2}\right) / \delta^{2}$, adjusting for unreliability: $n=n * / R$. Assuming a sample size of 100 per group, and $\rho$ (correlations between waves of data) $=0.5$, 0.6 and 0.7 , the resulting estimates of effect sizes are $\delta=4.21,3.77$ and 3.26 for ZBI and $\delta=3.21,2.87$ and 2.49 for GDS, thus demonstrating that a medium effect size (Cohen's $\mathrm{d}=0.33$ to 0.43 ) could be detected with this sample size.

\section{Power for sensitivity and descriptive effect modification analyses}

Assuming that the outcomes are correlated, power for MANCOVA was performed, taking into account possible baseline differences (by entering time) and adjusting for unreliability. Additionally, the calculations below assume that there is $20 \%$ attrition and missing data in some of the effect modifiers, for example, acculturation. We also modelled different correlations between the first and second waves of data, using the following formula: $\mathrm{R}_{\mathrm{xy}}^{2}=\mathrm{d}^{2} /\left(\mathrm{d}^{2}+1 / \mathrm{p}(1-\mathrm{p})\right)$, where $\mathrm{d}=\delta / \sigma$ and $\mathrm{p}$ is the proportion representing the allocation ratio $(\mathrm{p}=0.5$ for equal allocation to intervention and control groups) (Cohen, pp 490-3), $\quad \mathrm{f}^{2}=\mathrm{R}_{\mathrm{xy}}^{2} /\left(1-\mathrm{R}_{\mathrm{xy}}^{2}\right) \quad$ and $\mathrm{f}^{2}=\mathrm{f}^{2} \times \mathrm{R}_{\text {reliability }} \times 2(1-\rho)$ (adjusted for unreliability and $\rho)$. The non-centrality parameter is $\lambda=\mathrm{f}_{1}^{2}(\mathrm{u}+\mathrm{v}+1)$, where $\mathrm{u}=\mathrm{k}_{\mathrm{y}}$ (outcomes), and $\mathrm{v}=\mathrm{N}$ $-\mathrm{k}_{\mathrm{c}}-\mathrm{k}_{\mathrm{y}}-1 \quad\left(\mathrm{~N}=\right.$ total sample size and $\mathrm{k}_{\mathrm{c}}=$ covariates, eg, acculturation and type of care and their interactions). The effect sizes were obtained iteratively, based on the assumptions shown in table 1. Power for MANCOVA was also examined under several scenarios regarding the non-centrality parameter. The resulting $\lambda$ s are shown in table 2. The following assumptions were made: $\alpha=0.05$, $\sigma=9.8$ (ZBI) and 7.47 (GDS), reliability $=0.85, \delta=5$ (ZBI) and 4 (GDS) point reduction in the intervention relative to the control group.

Cohen's d ranged from 0.33 to 0.55 (see table 3) or between 3 and 5 points on the ZBI and 2.5 and 4 points on GDS end point means, roughly equivalent to a $0.3-$ $0.5 \mathrm{SD}$ end point difference in means, a moderate effect size for the primary analyses, assuming 100 per group.

Table 1 Assumptions for power calculations and effect sizes for MANCOVA

MANCOVA Assumptions: $\sigma=9.8(\mathrm{ZBI}), 7.47$

(GDS), $\alpha=0.05, R$ (reliability) $=0.85 ; g=2$

groups, $1-\beta=0.80$ ), $M=80 /$ group, 2 outcomes

and 3 covariates (possible effect modifiers)

$r$ (correlation between waves) $=0.5$

$r=0.6$

$r=0.7$

GDS, Geriatric Depression Scale; ZBI,Zarit Burden Interview.
ZBI point reduction in the intervention relative to control group ( $\delta$ )

5.39 (Cohen $d=0.55$ )

4.80 (Cohen $d=0.49$ )

4.21 (Cohen $d=0.43$ )
GDS point reduction in the intervention relative to the control group $(\delta)$

4.11 (Cohen $d=0.55$ )

3.66 (Cohen $d=0.49$ )

3.21 (Cohen $d=0.43$ ) 
Power for Zarit

( $\delta$ is the point reduction in th intervention relative to th control group)

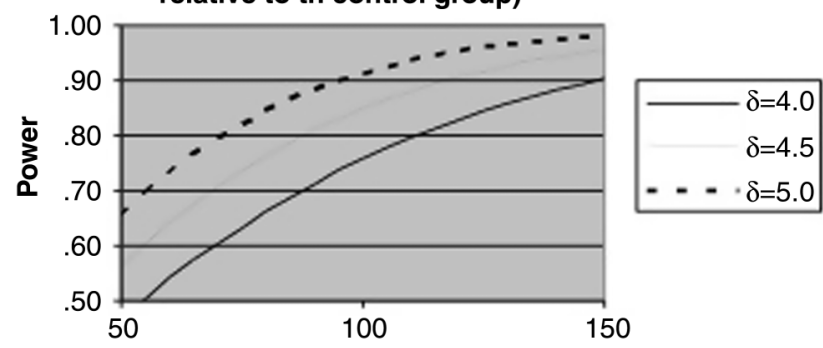

M(sample size per group)

Figure 1 Power for examining end point differences in the Zarit burden index outcome.

\section{Power for GDS}

( $\delta$ is the point reduction in th intervention relative to th control group)

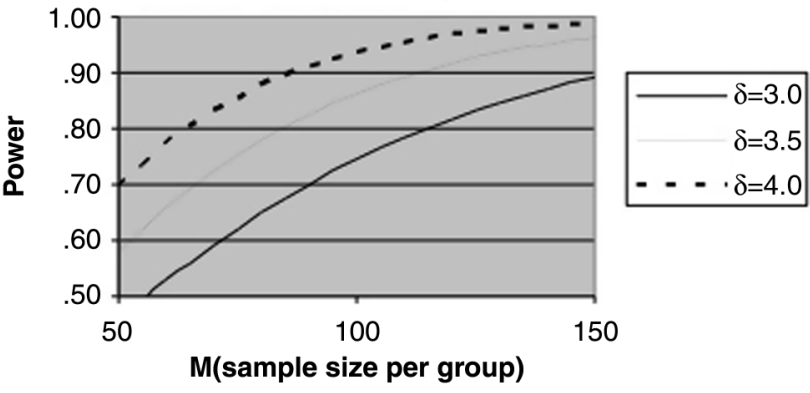

Figure 2 Power for examining end point differences in the GDS primary outcome. GDS, Geriatric Depression Scale.

Table 2 Power for MANCOVA under several non-centrality parameter scenarios

\begin{tabular}{llcc}
\hline (80/group) & $\boldsymbol{\rho}=\mathbf{0 . 5}$ & $\boldsymbol{\rho}=\mathbf{0 . 6}$ & $\boldsymbol{\rho}=\mathbf{0 . 7}$ \\
\hline $\mathrm{f}_{1}^{2}$ & 0.055 & 0.069 & 0.092 \\
$\Lambda$ & 8.85 & 11.06 & 14.75 \\
\hline
\end{tabular}

Table 3 Summary of effect sizes for different approaches to power calculations

Method

Effect size $(\Delta / \sigma)$

Two groups (Fleiss)

Two groups, different $\rho$ 0.43 $0.33-0.43$

MANCOVA ( 2 groups, $\rho=0.5,0.6,0.7$ )

$0.55,0.49,0.43$

End point differences and MANCOVA effect sizes under different assumptions about the correlation between baseline and end point mean values of the outcomes.

Conservatively, under the assumptions specified above regarding attrition and inclusion of potential effect modifiers, 80 participants per group will provide power $\geq 0.80$ to detect a 4 to 5 unit differential change in depression and burden. This is based on testing the timexgroup interaction, entering acculturation, type of caregiver (spouse, daughter) and the interaction of group by these terms in a MANCOVA, allowing for heterogeneous variances and modelling correlations between measures and over time. Even if the pooled variance is higher than assumed, medium effect sizes are still detectable. Thus, $80-100$ participants per arm will provide sufficient power to detect the hypothesised difference between the two study arms.

The study is monitored by a three member Data Safety and Monitoring Board (DSMB) that is convened twice a year. The DSMB comprised an expert in clinical trials of behavioural interventions, a neurologist with expertise in dementia and mental health and a psychiatrist. The DSMB is provided with up-to-date recruitment and adverse events data.

\section{DISSEMINATION}

The results of the study will be submitted for publication in a peer review journal. In addition, we will submit manuscripts on the characteristics of Hispanics in New York City once recruitment is completed. The funding agency will have no role in the content of these manuscripts.

\section{Author affiliations}

${ }^{1}$ Departments of Medicine and Epidemiology, Columbia University Medical Center, New York, New York, USA

${ }^{2}$ Burgio Geriatric Consulting, Tuscaloosa, Alabama, USA

${ }^{3}$ Departments of Psychiatry and Rehabilitation Medicine, New York University

School of Medicine, New York, New York, USA

${ }^{4}$ Riverstone Senior Life Services, New York, New York, USA

${ }^{5}$ CaringKind, New York, New York, USA

${ }^{6}$ Research Division, Hebrew Home at Riverdale, Bronx, New York, USA

${ }^{7}$ Weill Cornell Medical College, New York, New York, USA

Acknowledgements The authors express their gratitude to CaringKind, formerly known as the Alzheimer's Association, New York City Chapter, the Washington Heights-Inwood Council on Aging, New York Presbyterian Hospital and the Research Division of the Hebrew Home for the Aged for supporting the outreach and recruitment activities related to this project. They also express their gratitude to the busy caregivers who are participating in this study despite the enormous burden of caring for their loved one with dementia.

Contributors JAL is the study principal investigator and was responsible for obtaining funding, designing the study and drafting this manuscript; LB is a study co-principal investigator and was responsible for co-designing the study, designing the REACH implementation and drafting this manuscript. MM is a study co-principal investigator and was responsible for co-designing the study, designing the NYUCI implementation and drafting this manuscript; ID is the executive director of our community-based research partner Riverstone Senior Life Services and reviewed and approved the manuscript for content. JAL is the Executive Vice President and Director of programmes and services at our community research partner, Caringkind, formerly the Alzheimer's Association, New York City Chapter. MR and SS were responsible for the design of study logistics and questionnaires; JAT is the leader of the data-coordinating centre and was responsible for the overall study design, the statistical plan and drafting of this manuscript. JK assisted JAT with the development of the statistical plan. All authors read and approved the final manuscript.

Funding The primary source for the funding of this project is PCORI contract CE-1304-7160. Partial support for participant assessments is provided by the Irving Institute of Clinical Translational Research at Columbia University Medical Center, funded by a Clinical Translational Science Award (UL1 RR024156). 
Disclaimer The content is solely the responsibility of the authors and does necessarily represent the official views of funding agency.

Competing interests MM is the developer of the NYUCl and has received consulting fees for training providers. She is currently working on a Small Business Innovation Research grant to develop online training for the NYUCI. It is possible that MM will benefit in the future from the distribution of NYUC training materials. LB is the developer of the REACH OUT caregiver support program and has received consulting fees for training providers. He is currently working with Oxford University Press to publish a version of the REACH OUT training manual. It is possible that LB will benefit in the future from the distribution of REACH OUT training materials. The other author(s) declare that they have no competing interests.

Ethics approval Institutional Review Board of Columbia University Medical Center

Provenance and peer review Not commissioned; peer reviewed for ethical and funding approval prior to submission.

Open Access This is an Open Access article distributed in accordance with the Creative Commons Attribution Non Commercial (CC BY-NC 4.0) license, which permits others to distribute, remix, adapt, build upon this work noncommercially, and license their derivative works on different terms, provided the original work is properly cited and the use is non-commercial. See: http:// creativecommons.org/licenses/by-nc/4.0/

\section{REFERENCES}

1. Diagnostic and Statistical Manual of Mental Disorders, (DSM-IV). Washington (DC): American Psychiatric association, 1994.

2. Cummings JL. Alzheimer's Disease. N Engl J Med 2004;351:56-67.

3. Alzheimer's Association. 2011 Alzheimer's disease facts and figures. Alzheimers Dement 2011;7:208-44.

4. Chui HC, Mack W, Jackson JE, et al. Clinical criteria for the diagnosis of vascular dementia: a multicenter study of comparability and interrater reliability. Arch Neurol 2000;57:191-6.

5. Seshadri S, Wolf PA, Beiser A, et al. Lifetime risk of dementia and Alzheimer's disease. The impact of mortality on risk estimates in the Framingham Study. Neurology 1997;49:1498-504.

6. Evans DA, Funkenstein $\mathrm{HH}$, Albert MS, et al. Prevalence of Alzheimer's disease in a community population of older persons. Higher than previously reported. JAMA 1989;262:2551-6.

7. Hurd MD, Martorell P, Delavande A, et al. Monetary costs of dementia in the United States. N Engl J Med 2013;368: 1326-34.

8. Daviglus ML, Bell CC, Berrettini W, et al. NIH state-of-the-science conference statement: Preventing Alzheimer's disease and cognitive decline. NIH Consens State Sci Statements 2010;27:1-30.

9. Fagan T. Clinical Trials of Intravenous Bapineuzumab Halted. Alzforum 2012. http://www.alzforum.org/news/research-news/clinicaltrials-intravenous-bapineuzumab-halted

10. Humes KR, Jones NA, Ramirez RR. Overview of race and Hispanic origin: 2010. In: US Census Bureau, ed. 2010 census briefs. Washington (DC): US Department of Commerce, 2011.

11. Steffens DC, MacFall JR, Payne ME, et al. Grey-matter lesions and dementia. Lancet 2000;356:1686-7.

12. Noble JM, Manly JJ, Schupf N, et al. Type 2 diabetes and ethnic disparities in cognitive impairment. Ethn Dis 2012;22:38-44.

13. Tang MX, Stern Y, Marder K, et al. The APOE-epsilon4 allele and the risk of Alzheimer disease among African Americans, whites, and Hispanics. JAMA 1998;279:751-5.

14. Lyketsos CG, Steele C, Baker L, et al. Major and minor depression in Alzheimer's disease: prevalence and impact. $J$ Neuropsychiatry Clin Neurosci 1997:9:556-61.

15. Lyketsos CG, Sheppard JM, Steinberg M, et al. Neuropsychiatric disturbance in Alzheimer's disease clusters into three groups: the Cache County study. Int J Geriatr Psychiatry 2001;16:1043-53.

16. Lyketsos CG, Lopez O, Jones B, et al. Prevalence of neuropsychiatric symptoms in dementia and mild cognitive impairment: results from the cardiovascular health study. JAMA 2002;288:1475-83.

17. Monin JK, Schulz R. Interpersonal effects of suffering in older adult caregiving relationships. Psychol Aging 2009;24:681-95.

18. Yaffe K, Fox P, Newcomer R, et al. Patient and caregiver characteristics and nursing home placement in patients with dementia. JAMA 2002;287:2090-7.
19. Taylor DH, Jr., Ezell M, Kuchibhatla M, et al. Identifying trajectories of depressive symptoms for women caring for their husbands with dementia. J Am Geriatr Soc 2008;56:322-7.

20. Covinsky KE, Newcomer R, Fox P, et al. Patient and caregiver characteristics associated with depression in caregivers of patients with dementia. J Gen Intern Med 2003;18:1006-14.

21. Port CL, Zimmerman S, Williams $\mathrm{CS}$, et al. Families filling the gap: comparing family involvement for assisted living and nursing home residents with dementia. Gerontologist 2005;45:87-95.

22. Schulz R, Belle SH, Czaja SJ, et al. Long-term care placement of dementia patients and caregiver health and well-being. JAMA 2004;292:961-7.

23. Gelman CR. Learning from recruitment challenges: Barriers to diagnosis, treatment, and research participation for Latinos with symptoms of Alzheimer's disease. J Gerontol Soc Work 2010;53:94-113.

24. Cox C, Monk A. Strain among caregivers: comparing the experiences of African American and Hispanic caregivers of Alzheimer's relatives. Int J Aging Hum Dev 1996;43:93-105.

25. Valle R, Yamada AM, Barrio C. Ethnic differences in social network help-seeking strategies among Latino and Euro-American dementia caregivers. Aging Ment Health 2004;8:535-43.

26. Ranney MJ, Aranda MP. Factors associated with depressive symptoms among Latino family dementia caregivers. J Ethn Cult Divers Soc Work 2001;10:1-21.

27. Coon DW, Rubert M, Solano N, et al. Well-being, appraisal, and coping in Latina and Caucasian female dementia caregivers: findings from the REACH study. Aging Ment Health 2004;8:330-45.

28. Mittelman MS, Roth DL, Clay OJ, et al. Preserving health of Alzheimer caregivers: impact of a spouse caregiver intervention. Am J Geriatr Psychiatry 2007;15:780-9.

29. Mittelman MS, Roth DL, Coon DW, et al. Sustained benefit of supportive intervention for depressive symptoms in caregivers of patients with Alzheimer's disease. Am J Psychiatry 2004;161:850-6.

30. Mittelman MS, Brodaty $\mathrm{H}$, Wallen AS, et al. A three-country randomized controlled trial of a psychosocial intervention for caregivers combined with pharmacological treatment for patients with Alzheimer disease: effects on caregiver depression.

Am J Geriatr Psychiatry 2008;16:893-904.

31. Belle SH, Burgio L, Burns R, et al. Enhancing the quality of life of dementia caregivers from different ethnic or racial groups: a randomized, controlled trial. Ann Intern Med 2006;145:727-38.

32. Alzheimer's Association. 2013 Alzheimer's disease facts and figures. Alzheimers Dement 2013;9:208-45.

33. Gaugler JE, Reese M, Mittelman MS. Effects of the NYU caregiver intervention-adult child on residential care placement. Gerontologist 2013:53:985-97.

34. Pearlin LI, Mullan JT, Semple SJ, et al. Caregiving and the stress process: an overview of concepts and their measures. Gerontologist 1990;30:583-94.

35. Mittelman MS, Roth DL, Haley WE, et al. Effects of a caregiver intervention on negative caregiver appraisals of behavior problems in patients with Alzheimer's disease: results of a randomized trial. $J$ Gerontol B Psychol Sci Soc Sci 2004;59:P27-34.

36. Mittelman MS, Haley WE, Clay OJ, et al. Improving caregiver well-being delays nursing home placement of patients with Alzheimer disease. Neurology 2006:67:1592-9.

37. Roth DL, Mittelman MS, Clay OJ, et al. Changes in social support as mediators of the impact of a psychosocial intervention for spouse caregivers of persons with Alzheimer's disease. Psychol Aging 2005;20:634-44.

38. Schulz R, Burgio L, Burns R, et al. Resources for Enhancing Alzheimer's Caregiver Health (REACH): overview, site-specific outcomes, and future directions. Gerontologist 2003;43:514-20.

39. Belle SH, Czaja SJ, Schulz R, et al. Using a new taxonomy to combine the uncombinable: integrating results across diverse interventions. Psychol Aging 2003;18:396-405.

40. Burgio L, Stevens A, Guy D, et al. Impact of two psychosocial interventions on White and African American family caregivers of individuals with dementia. Gerontologist 2003:43:568-79.

41. Bourgeois MS, Burgio LD, Schulz R, et al. Modifying repetitive verbalizations of community-dwelling patients with $\mathrm{AD}$. Gerontologist 1997;37:30-9.

42. Napoles AM, Chadiha L, Eversley R, et al. Reviews: developing culturally sensitive dementia caregiver interventions: are we there yet? Am J Alzheimers Dis Other Demen 2010;25:389-406.

43. Van Mierlo LD, Meiland FJ, Van der Roest HG, et al. Personalised caregiver support: effectiveness of psychosocial interventions in subgroups of caregivers of people with dementia. Int J Geriatr Psychiatry 2012;27:1-14. 
44. Helfand M, Berg A, Flum D, et al. Patient Centered Outcomes Research Institute Draft Methodology Report: "Our Questions, Our Decisions: Standards for Patient-centered Outcomes Research. I Washington, DC.: Patient Centered Outcomes Research Institute, 2012:1-192.

45. Kieser M, Friede T. Planning and analysis of three-arm non-inferiority trials with binary endpoints. Stat Med 2007;26: 253-73

46. Berger ML, Dreyer N, Anderson F, et al. Prospective observational studies to assess comparative effectiveness: the ISPOR good research practices task force report. Value Health 2012;15:217-30.

47. Petkova E, Teresi J. Some statistical issues in the analyses of data from longitudinal studies of elderly chronic care populations. Psychosom Med 2002;64:531-47.

48. Hernán MA, Hernández-Díaz S. Beyond the intention-to-treat in comparative effectiveness research. Clin Trials 2012;9:48-55.

49. Luchsinger JA, Tipiani D, Torres-Patiño $G$, et al. Characteristics and mental health of hispanic dementia caregivers in New York City. Am J Alzheimers Dis Other Demen 2015;30:584-90.

50. Fitzgerald JF, Smith DM, Martin DK, et al. Replication of the multidimensionality of activities of daily living. J Gerontol 1993;48: S28-31.

51. Bruce B, Fries JF, Ambrosini D, et al. Better assessment of physical function: item improvement is neglected but essential. Arthritis Res Ther 2009;11:R191.

52. Rose M, Bjorner JB, Becker J, et al. Evaluation of a preliminary physical function item bank supported the expected advantages of the Patient-Reported Outcomes Measurement Information System (PROMIS). J Clin Epidemiol 2008;61:17-33.

53. Fries JF, Krishnan E, Rose M, et al. Improved responsiveness and reduced sample size requirements of PROMIS physical function scales with item response theory. Arthritis Res Ther 2011;13:R147.

54. Cohen S, Kamarck T, Mermelstein R. A global measure of perceived stress. J Health Soc Behav 1983:24:385-96.

55. Teri L, Truax P, Logsdon R, et al. Assessment of behavioral problems in dementia: the revised memory and behavior problems checklist. Psychol Aging 1992;7:622-31.

56. Stokes JP. Toward an understanding of cohesion in personal change groups. Int J Group Psychother 1983;33:449-67.

57. Reisberg B, Ferris SH, de Leon MJ, et al. The Global Deterioration Scale for assessment of primary degenerative dementia. Am J Psychiatry 1982;139:1136-9.

58. Teresi JA, Ramirez M, Lai JS, et al. Occurrences and sources of differential item functioning (DIF) in patient-reported outcome measures: description of DIF methods, and review of measures of depression, quality of life and general health. Psychol Sci $Q$ 2008;50:538.

59. Radloff $L$. The CES-D scale: a self report depression scale for research in the general population. Appl Psychol Meas 1977:385-401.
60. Yesavage JA, Brink TL, Rose TL, et al. Development and validation of a geriatric depression screening scale: a preliminary report. J Psychiatr Res 1982;17:37-49.

61. Grayson DA, Mackinnon A, Jorm AF, et al. Item bias in the Center for Epidemiologic Studies Depression Scale: effects of physical disorders and disability in an elderly community sample. $J$ Gerontol B Psychol Sci Soc Sci 2000;55:P273-82.

62. Reeve BB. Special issues for building computerized-adaptive tests for measuring patient-reported outcomes: The National Institute of Health's investment in new technology. Med Care 2006;44(Suppl 3): S198-204.

63. Reeve BB, Hays RD, Bjorner JB, et al. Psychometric evaluation and calibration of health-related quality of life item banks: plans for the Patient-Reported Outcomes Measurement Information System (PROMIS). Med Care 2007;45(Suppl 1):S22-31.

64. Teresi JA, Ocepek-Welikson K, Kleinman M, et al. Analysis of differential item functioning in the depression item bank from the Patient Reported Outcome Measurement Information System (PROMIS): An item response theory approach. Psychol Sci $Q$ 2009;51:148-80.

65. Teresi JA, Ocepek-Welikson K, Kleinman M, et al. Psychometric properties and performance of the Patient Reported Outcomes Measurement Information System $®$ (PROMIS $®$ ) depression short forms in ethnically diverse groups. Psychol Test Assess Model 2016;58:141-81.

66. Gort AM, Mingot M, Gomez X, et al. Use of the Zarit Scale for assessing caregiver burden and collapse in caregiving at home in dementias. Int J Geriatr Psychiatry 2007;22:957-62.

67. Wisniewski SR, Belle SH, Coon DW, et al. The Resources for Enhancing Alzheimer's Caregiver Health (REACH): project design and baseline characteristics. Psychol Aging 2003:18:375-84

68. Elliott AF, Burgio LD, Decoster J. Enhancing caregiver health: findings from the resources for enhancing Alzheimer's caregiver health II intervention. J Am Geriatr Soc 2010;58:30-7.

69. Marín G, Posner SF. The role of gender and acculturation on determining the consumption of alcoholic beverages among Mexican-Americans and Central Americans in the United States. Int J Addict 1995;30:779-94.

70. Robins JM. Correction for non-compliance in equivalence trials. Stat Med 1998;17:269-302; discussion 87-9.

71. Diggle PJ, Liang KY, Zeger SL. Analysis of longitudinal data. New York: Oxford University Press, 1994.

72. Cohen J. Statistical power analysis for the behavioral sciences. New York: Academic Press, 1977:1988.

73. Fleiss JL. Design and Analysis of Clinical Experiments. New York: John Wiley and Sons, 1999.

74. Overall JE, Doyle SR. Estimating sample sizes for repeated measurement designs. Control Clin Trials 1994;15:100-23. 


\section{Correction}

Luchsinger JA, Burgio L, Mittelman M, et al. Northern Manhattan Hispanic Caregiver Intervention Effectiveness Study: protocol of a pragmatic randomised trial comparing the effectiveness of two established interventions for informal caregivers of persons with dementia. BMJ Open 2016;6:e014082.

The funding statement is not completed, it should read: "Funding Research reported in this protocol has been funded through a Patient-Centered Outcomes Research Institute (PCORI) Award (CE-1304-7160). The report in this protocol is solely the responsibility of the authors and does not necessarily represent the views of the Patient-Centered Outcomes Research Institute (PCORI), its Board of Governors or Methodology Committee. Partial support for this study is provided by the Irving Institute for Clinical Translational Research at Columbia University Medical Center, funded by a Clinical Translational Science Award (UL1 RR024156), and by grant 4R01NR014430, from the National Institutes of Health".

Open Access This is an Open Access article distributed in accordance with the Creative Commons Attribution Non Commercial (CC BY-NC 4.0) license, which permits others to distribute, remix, adapt, build upon this work noncommercially, and license their derivative works on different terms, provided the original work is properly cited and the use is non-commercial. See: http://creativecommons.org/licenses/by-nc/4.0/

BMJ Open 2016;6:014082corr1. doi:10.1136/bmjopen-2016-014082corr1

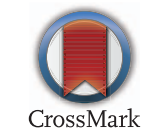

